\title{
Aportes del concepto de interpenetración a los debates de las políticas públicas en América Latina
}

\author{
Contributions of the Concept of Interpenetration to Discussions on Public Policies in Latin \\ America
}

Teresa Matus

Departamento de Trabajo Social, Universidad de Chile, Chile

RESUMEN

El presente trabajo propone una innovación teórica en el ámbito de la intervención social y las políticas públicas en América Latina a partir del concepto de interpenetración y su relación con una dialéctica negativa. En este sentido, se propone trazar puentes entre tradiciones de pensamiento sistémico y crítico para el abordaje de las políticas públicas en la región. El trabajo no solamente aborda las dimensiones conceptuales de este aporte sino también problemas prácticos de este nuevo enfoque.

PALABRAS ClaVE: Diferenciación funcional; Interpenetración; Políticas públicas; Pensamiento negativo; América Latina

\section{ABSTRACT}

This paper proposes a theoretical innovation in the field of social intervention and public policies in Latin America based on the concept of interpenetration and its relation to negative dialectic. In this sense, it is proposed to build bridges between traditions of systemic and critical thought for addressing public policy in the region. The paper not only addresses the conceptual dimensions of this contribution but also practical problems of this new approach.

KEYWORDS: Functional differentiation; Interpenetration; Public policies; Negative thinking; Latin America

\section{INTRODUCCIÓN}

Existen aportes específicos desde una teoría de sistemas contextual que iluminan rupturas con un pensamiento clásico de teorías de la diferenciación: "para connotar las relaciones de ruptura con la tradición, es indispensable explicar en qué consiste la diferencia entre esto y lo nuevo, para poder encontrar las posibilidades de su trasposición" (Luhmann 1998: 199). Mostrando una línea de innovación, se desarrolla el concepto de interpenetración como un horizonte de inter-referencia para observar ciertas dimensiones de la lógica de los debates sobre políticas públicas en América Latina. De allí que el artículo exponga: (1) un diagnóstico basado en diversas investigaciones de los debates sobre políticas públicas, espe-

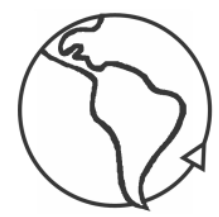

REVISTA MAD - UNIVERSIDAD DE CHILE

Revista del Magíster en Análisis Sistémico Aplicado a la Sociedad

Facultad de Ciencias Sociales, Departamento de Antropología

Universidad de Chile

ISSN 0718-0527

www.revistamad.uchile.cl 
cialmente referido a algunas propuestas sociales de los últimos 25 años en América Latina, ciertas lógicas imperantes y la forma en que desde ellas se observan las brechas, fragmentaciones, duplicidades y falta de coordinación. Lo anterior se cruza con una comparación realizada en los gobiernos de tres mandatarias latinoamericanas actuales: Dilma Rousseff, Cristina Fernandez y Michelle Bachelet. (2) Una raíz diversa para la observación entre sistemas, cuyos antecedentes emergen en el concepto de penetración de Georg W. F. Hegel y, mostrando aprecio por el debate entre la tradición frankfurteana y funcional, en la noción de yuxtaposición en Theodor W. Adorno. (3) el giro del concepto de interpenetración en Niklas Luhmann cuyos rasgos claves son: entender que se "trata de un entorno especial de los sistemas sociales: el de los seres humanos y sus relaciones con los sistemas sociales" (Luhmann 1998: 203), un sistema de disposición recíproca, donde se ejerce selectividad y se impulsa la capacidad de conexión de diverso orden. Se trata de una relación inter-sistémica donde la penetración es un sistema que pone a disposición su propia complejidad y con ello indeterminación, contingencia y coacción de selección para construir otro sistema. Ahora bien, hay interpenetración cuando esta situación es recíproca; es decir, cuando ambos sistemas se posibilitan mutuamente aportar al otro su propia complejidad pre-construida. El concepto de interpenetración, de este modo, sustituye las doctrinas del derecho natural, así como los intentos sociológicos que han trabajado con los conceptos de necesidad y las teorías de roles y de socialización. (4) Tomando esos antecedentes, se muestra qué pasa si se desarrollan algunas dimensiones de la noción de interpenetración y cómo podría girar la lógica de los debates en las políticas públicas en América Latina buscando, desde un Luhmann controversial, abierto y negativo, pasar de "conceptualizaciones sin esperanza a conceptualizaciones improbables".

\section{TENSIONES EN LA LÓGICA DE LAS POLÍTICAS PÚBLICAS EN AMÉRICA LATINA}

En las investigaciones sobre políticas públicas de los últimos 25 años encontramos fuertes nudos críticos referidos por lo menos a los siguientes tópicos: pasar de una focalización a criterios más universales en las políticas sociales, un cuestionamiento a los sistemas de transferencia de renta como mecanismos únicos de mejoramiento social, una hegemonía de evaluación de las políticas públicas observadas desde la tipificación de la demanda, una naturalización con diversos mecanismos de la antinomia: cre- 
cimiento/distribución social. Ya en la última década del siglo XX existe una separación del financiamiento de la provisión pública de servicios sociales, una introducción de competencia en la producción de servicios a través de agencias independientes y la creación de redes de protección social con programas de transferencias no contributorias focalizadas en "los pobres y personas vulnerables" que, según la CEPAL, condiciona fuertemente el horizonte de cambio social en el continente. Estas iniciativas consideran a personas en condiciones de pobreza crónica y aquellos en riesgo debido a causas exógenas. Se enfrentan con programas de asistencia social entre los que se incluyen: transferencias monetarias, programas alimentarios, subsidios básicos de precios a algunos productos, programas de obras públicas menores. A ellos el Banco Mundial en el 1997 los denomina: "Safety net programs and poverty reduction". Una década después, Cohen y Franco (2006) resaltan aspectos positivos de esta lógica ya que según ellos: permitió experimentar soluciones, normas y procedimientos flexibles y fomentó la presencia de nuevos actores. Sin embargo, el propio BID va a sostener que esas medidas:

Se concibieron como programas de protección temporales y anti cíclicos que proporcionarían ingreso y empleo adicionales a las víctimas de las crisis. Sin embargo, en la práctica no sirvieron en absoluto como redes de protección; más bien se convirtieron en instituciones permanentes especializadas en la prestación de servicios sociales a las comunidades pobres. (BID 2008).

Según Claudia Serrano (2005), una comparación posible de transferencias condicionadas es la siguiente:

TABLA 1. TRANSFERENCIAS CONDICIONADAS

\begin{tabular}{|l|l|l|}
\hline Brasil & $\begin{array}{l}- \text { Hambre Cero } \\
- \text { Bolsa Familia }\end{array}$ & $\begin{array}{l}- \text { Bono escolar } \\
- \text { Bono Alimentación }\end{array}$ \\
\hline Argentina & - Jefes y Jefas de hogar & - Pago ayuda económica \\
\hline Chile & - Chile Solidario & $\begin{array}{l}- \text { Apoyo Psicosocial } \\
- \text { Bono Protección } \\
-\begin{array}{l}\text { Acceso preferente a re- } \\
\text { des públicas }\end{array}\end{array}$ \\
\hline
\end{tabular}

FUENTE: Serrano (2005)

Para Serrano se entiende la protección social como: 
La labor del Estado orientada a apoyar a las personas que enfrentan vulnerabilidad y riesgo social, lo que dificulta sus posibilidades de integración social. En términos concretos está constituida por el conjunto de transferencias y prestaciones no contributivas distintas a las prestaciones continuas de los servicios sociales básicos. (Serrano 2005: 7)

Uno de los principales nudos críticos de lo anterior, es la lógica de la focalización: la focalización es un medio para mejorar la eficiencia de un programa a través de incrementar los beneficios que pueden recibir los más pobres en el contexto de un presupuesto fijo. Sin embargo, esto supone un horizonte temporal que, medido en Chile en el 2009, daba una intersección posible entre demanda y oferta acumulada de 24 años.

FIGURA 1. CHILE: OFERTA Y DEMANDA DE PROGRAMAS EN 24 AÑOS

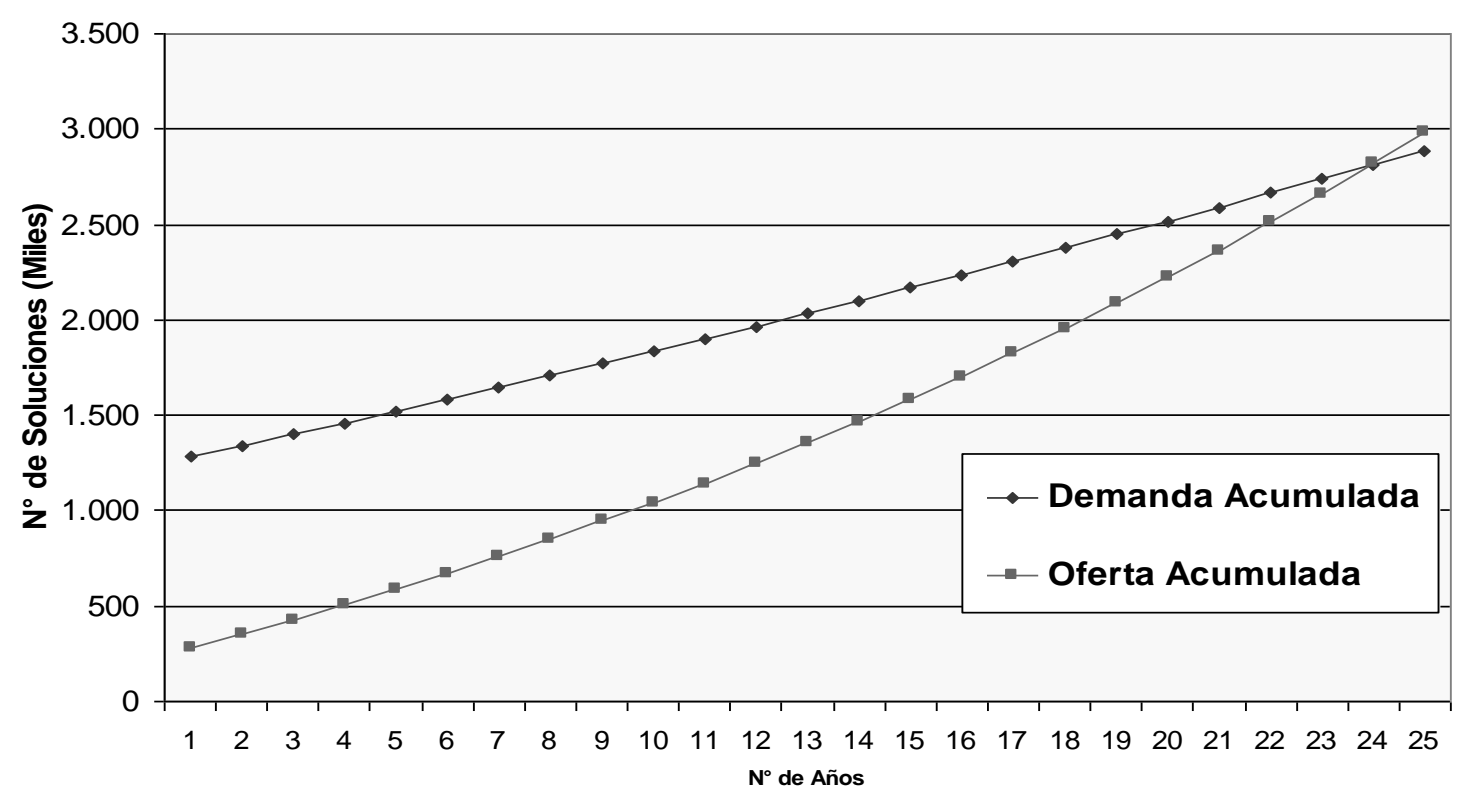

FUENTE: Irarrázabal (2009)

Lo que existe es desfocalización. Según Coady, Grosh y Hoddinott (2004) La focalización puede funcionar pero no siempre, ya que un cuarto de los programas son regresivos, no hay un método claramente preferido de observación y evaluación y existe un débil desarrollo de ranking y sistemas de información. De allí que surjan en América Latina, distintos esfuerzos por el registro y la medición: 
TABLA 2. SISTEMAS DE REGISTRO Y MEDICIÓN DE FOCALIZACIÓN

\begin{tabular}{|c|c|c|c|c|c|c|}
\hline Componentes & Argentina & Brasil & Colombia & Costa Rica & Chile & México \\
\hline $\begin{array}{l}\text { Registro } \\
\text { Único } \\
\text { Beneficiarios } \\
\text { (RUB) }\end{array}$ & 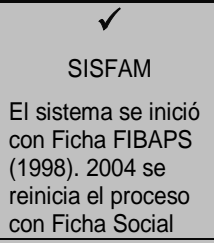 & $\begin{array}{c}\sqrt{ } \\
\text { CADUNICO }\end{array}$ & \begin{tabular}{l}
\multicolumn{1}{c}{$\boldsymbol{l}$} \\
$\qquad$ SISBEN \\
Iniciado 1993. \\
Nuevo SISBEN a \\
partir 2003.
\end{tabular} & $\begin{array}{c}\checkmark \\
\text { SIPO } \\
\text { Iniciado en } 1991 \\
\text { como SISBEN }\end{array}$ & \begin{tabular}{l}
$\quad \checkmark$ \\
\multicolumn{1}{c}{$\quad$} \\
Sistema CAS \\
Iniciado en 1979, \\
ha tenido tres \\
modificaciones \\
importantes
\end{tabular} & $\begin{array}{c}\checkmark \\
\text { Padrón de } \\
\text { Beneficiarios } \\
\text { Iniciado } 2001\end{array}$ \\
\hline $\begin{array}{l}\text { Índice de } \\
\text { Focalización } \\
\text { de } \\
\text { Beneficiarios } \\
\text { (IFB) }\end{array}$ & $\begin{array}{c}\text { Indice de Calidad } \\
\text { de Vida ICV } \\
\text { (Aplicación parcial) }\end{array}$ & $\begin{array}{c}\text { व } \checkmark \\
\text { Focalización en } \\
\text { base a ingresos } \\
\text { autoreportados }\end{array}$ & SISBEN & Puntaje SIPO & Puntaje CAS & $\begin{array}{c}\checkmark \\
\text { Clasificación de } \\
\text { beneficiarios a } \\
\text { partir de una } \\
\text { ecuación } \\
\text { discriminante } \\
\end{array}$ \\
\hline $\begin{array}{l}\text { Sistema de } \\
\text { Integración de } \\
\text { Bases de } \\
\text { Datos. } \\
\text { (SIBD) }\end{array}$ & $\begin{array}{l}\quad \checkmark \\
\text { Sistema de } \\
\text { Identificación } \\
\text { Nacional Tributario } \\
\text { y Social SINTYS } \\
(1999)\end{array}$ & $\begin{array}{l}\quad \text { Integración parcial } \\
\text { con bases de } \\
\text { programas } \\
\text { usuarios. }\end{array}$ & $\begin{array}{l}\text { Cruces parciales ad } \\
\text { hoc }\end{array}$ & \begin{tabular}{l}
\multicolumn{1}{c}{ व } \\
Integración parcial \\
con bases de \\
identificación y Seg. \\
Social
\end{tabular} & \begin{tabular}{l}
\multicolumn{1}{c}{$\boldsymbol{x}$} \\
No hay sistema de \\
integración. \\
A partir 2005, \\
Sistema Integrado
\end{tabular} & $\begin{array}{l}\text { Confronta de } \\
\text { padrones sociales }\end{array}$ \\
\hline
\end{tabular}

FUENTE: Elaboración propia

Lo que se propone es que la lógica anterior llegó a un punto tal de saturación que sus explicaciones sobre brechas, fragmentaciones, duplicidades y falta de coordinación, fueron respondidas por los sistemas políticos de algunos países latinoamericanos con programas que aun radicalizando los sistemas de apoyo social, los giraban fuertemente en su contenido, pensándolos desde otra matriz. Esto es lo que podemos observar al menos en tres mandatarias latinoamericanas: Cristina Fernandez, Dilma Rousseff y Michelle Bachelet. Ahora bien, la temeridad de su desafío a los dioses y a robar el fuego sagrado de la lógica económica transformada en una teodicea de mercado para enfrentar la dicotomía naturalizada de distribución, reconocimiento versus crecimiento económico, las tiene paradojalmente en la cuerda floja, después de llegar a la presidencia con altos niveles de aprobación pública. Entender estos fenómenos y analizar los aportes de un concepto como el de interpenetración es la referencia de este escrito. En el último año, hemos visto descender los porcentajes de adhesión a los gobiernos de Roussef en Brasil, Fernandez en Argentina y Bachelet en Chile en proporciones diversas pero con ruidos compartidos. Si se efectúa un análisis de estas tres mandatarias y sus gobiernos podemos observar que: 
1.1. TRANSGREDIR EL FUEGO DE LOS DIOSES Y ENFRENTAR AL SISTEMA NEOLIBERAL CONFIGURA NUEVOS ESCENARIOS

Ya Max Weber en su clásico texto de Economía y Sociedad (2002) sostiene la tesis de la teodicea del mercado y la supraposición de la economía. Asimismo Durkheim (2007) coloca la economía en la matriz de aquello sagrado que provoca, a la vez, fascinación y terror. En esos términos las tres mandatarias han jugado con fuego, queriendo como Ícaro arrebatar su fulgor a los dioses. Con sus activas agendas de reformas han tocado los tributos y las bases imponibles de impuestos, las asignaciones de filiación, la educación, la previsión social, las reformas de sistemas electorales; han atacado las raíces conservadoras proponiendo nuevas leyes civiles. Es decir, no sólo han penetrado en el recinto de la redistribución, sino también en el del reconocimiento.

En Chile, son cinco las reformas principales: La reforma educativa, la reforma tributaria, la reforma electoral, las reformas civiles (uniones civiles entre parejas del mismo sexo y ley de aborto), las reformas en la previsión social y salud

Por su parte Cristina Fernandez en Argentina destaca en sus obras de gobierno la Asignación Universal por Hijo, la restatización de los fondos jubilatorios, el programa Conectar Igualdad, el aumento en el presupuesto para ciencia e investigación, la Ley de Servicios de Comunicación Audiovisual, la Ley de matrimonio igualitario, la re estatización de la empresa Yacimientos Petrolíferos Fiscales (YPF) y la reforma del Banco Central, entre otras.

En Brasil, Dilma Rousseff ajustó el impuesto a la renta e incrementó la asignación del programa Bolsa Familia. A pesar del marco de restricciones económicas, ha existido una expansión de los planes sociales que le han permitido aplicar una serie de políticas inclusivas que significaron una caída sustancial de los indicadores relacionados con la pobreza, la salud y la educación.

Todo lo anterior configura un escenario clásico de descontentos: el rechazo de los sectores empresariales y conservadores del sistema neoliberal, que en la crónica de una muerte anunciada, pronostican y cumplen la predicción de una baja en el crecimiento económico y la afectación de los diversos sistemas de inversión. A lo anterior se le suma el descontento de diversos sectores sociales que ven como las reformas, en su proceso de regulación y configuración de leyes se van amoldando, reduciendo, limi- 
tando sus potenciales frutos de inclusión y cambio social. Los opositores a sus gobiernos argumentan que los cambios frenarán el crecimiento de la economía, que ya atraviesa una desaceleración. Es decir, se produce un sistema enunciativo que lee las reformas como crisis y que se comporta comunicativamente desde allí.

1.2. EL EFECTO DOMINÓ EN MATERIAS DE CORRUPCIÓN HABLA MÁS QUE DE UN "RUIDO COMUNICATIVO"

A lo anterior, se podría decir que cada mandataria afronta su propia cadena de corrupción: la Petrobras en Brasil, el caso Caval en Chile y la muerte del fiscal Nisman en Argentina.

En el escándalo de corrupción de Petrobras en Brasil ha involucrado altos directivos y políticos cercanos a la mandataria y ha repercutido en su relación con el parlamento. En Chile, los casos de corrupción política relacionados con las empresas Penta, Soquimich y Caval han hecho caer la popularidad de la mandataria a mínimos históricos. Finalmente, en Argentina, las aún oscuras circunstancias de la muerte del fiscal Alberto Nisman que se encontraba investigando a la presidenta Fernández, ha sembrado un manto de dudas acerca de la mandataria.

Todos estos casos han redundado en bajas considerables de popularidad para las mandatarias y diversos problemas en sus agendas y planes de gobierno.

Tres mandatos impactados por la corrupción. Aunque esto sea tan clásico como los reclamos de San Agustín tanto hacia la propia Roma, como hacia los bárbaros, es interesante ver que, aunque marcan descensos de popularidad, ya no estamos en el escenario ni en el mundo de $\mathrm{Ni}$ xon, al tener que renunciar por el entonces escándalo de Watergate. Es más, ya no se usa el prefijo ni se habla de Cavalgate, de Petrogate o de Nismangate. El impacto de Jen Baudrillard (1995) con su "transparencia del mal", la "banalidad del mal" denunciada con grandes costos por Hannah Arendt (1999), pareciera que ya no consiguen seguir la forma que adquiere la relación entre corrupción, dinero y política. Más bien, pudiese ser que el reconocimiento de su desdiferenciación es más bien transversal y que ello genera posibilidades nuevas de estabilidad/inestabilidad. 


\subsection{UNA ECONOMÍA EN DESCENSO PONE A PRUEBA LA INTERPENETRACIÓN DE} CRECIMIENTO Y ENFRENTAMIENTO DE LA DESIGUALDAD

El contexto que completa a estos tres gobiernos, lo brindan los datos de la economía. Todos estos países han bajado sus expectativas de crecimiento y han reducido sus metas de ahorro fiscal. En consecuencia, lo que las tres mandatarias ponen a prueba es una lógica incombustible en el ámbito económico y que es reproducida por los medios de prensa: la dicotomía entre crecimiento y desigualdad. Aunque hayan existido ya muchas investigaciones donde se muestra que un crecimiento con desigualdad no sólo es condenable moralmente sino que es de baja efectividad para la propia economía, hay una resistencia pertinaz que no retrocede.

Se podría hacer un enorme montón de textos combinados incluso de Kliksberg, Amartya Sen y Mohammad Yunus y pareciera que ya los datos escalofriantes de sus resultados y sus avisos en "El informe Kliksberg, Primero la Gente, América Latina frente a la crisis" resultan impotentes para movilizar a las políticas públicas hacia otra lógica.

La obra de Bernardo Kliksberg (2011) que revisa algunos de los principales escándalos éticos que sacuden el Siglo XXI es un ejemplo, entre otros, de esa suerte de sordera con que se opera a la hora de cuestionar políticas públicas que buscan generar otra relación entre crecimiento y desigualdad.

Por tanto, los logros sociales, el desplazamiento de la matriz focalizadora, las reformas civiles, las propuestas políticas, siguen siendo observadas por el ojo de una aguja econométrica comandada por la fatalidad del " $\mathrm{O}$ ", donde crecimiento y desigualdad están en lados adversos. Y aunque desde muchos sectores se reclama mayor efectividad y coordinación funcional, esta demanda no puede ser satisfecha porque no se cumple una de sus condiciones básicas de posibilidad: el desencadenar mecanismos de interpenetración. De allí la relevancia de adentrarse en su comprensión.

\section{APRECIAR LA RAÍZ DE UN PENSAMIENTO NEGATIVO}

La noción de interpenetración en la obra de Luhmann es un concepto altamente exigente, tanto en sus requerimientos de comprensión como, mucho más, si se busca recorrerlo, experimentarlo, pensar con él. No se encuentra, en consecuencia "a la mano". Por tanto, se precisa de un cierto entrenamiento, de una preparación, de una disposición, de una lógica que 
esté a la altura de ese desafío. De la misma forma que la noción de intersubjetividad en Husserl permanece cerrada si no existe un trabajo fenomenológico de las subjetividades involucradas. Acá es posible sostener que la relación sistémica y sus oportunidades comunicativas se fundan no sólo en lo que cada sistema selecciona sino en aquello que ya no se selecciona. De otro modo, nos acercamos a esas nociones con claves argumentales que paradojalmente nos devuelven a una concepción reducida, que nos expulsan de la potencialidad de la noción de interpenetración: volver a pensarla desde un cierto sujeto, colocar el foco en el sistema o en el espacio existente entre uno y otro sistema, pensar los nexos sistema/entorno desde una sola distinción.

Así, el argumento busca entrenar una clave, ese movimiento que ya Hegel denominaba: el trabajo del concepto. Recurrir a una forma de pensar existente en la tradición de la filosofía moderna, nos coloca en el prisma del flujo, del cambio, de esos fundamentos líquidos, de esa zona donde todo lo sólido se desvanece en el aire y que se enciende y se pone en marcha mediante un vértigo negativo. La negatividad, ese espíritu inquieto que todo lo niega, tiene expresiones que son posibles de afinar para distinguirlas: la refutación popperiana, la de-construcción foucaultiana, la reificación luckasiana, la ruina benjaminiana, la negatividad adorniana. Por tanto, podría pensarse que en la observación de segundo orden y en la base del contructivismo luhmanniano habita un movimiento deconstructivo, tan radical uno cuanto otro. Hacer un viaje por algunas de esas negatividades es lo que se propone emprender al enunciar: penetración, yuxtaposición, interpenetración.

En primer lugar, cuando Hegel hace emerger el concepto de penetración en su Ciencia de la Lógica, lo coloca al interior de los trabajos negativos (Hegel 1969: 78). Es decir, pone exigencias que radican, entre otras, en las siguientes:

- Entender que la penetración no es el paso de uno a otro sino que en el paso se encuentra la verdad en el otro, porque toca su propio fondo.

- De este modo, esa relación entre palabras y cosas, entre el acto y su praxis, se encuentra más allá de sí, se funda en la relación, en la unidad concreta y múltiple de pensamiento y acto. Más allá del nombre y en su propia anulación, para poder pensarse como relación. 
- En consecuencia, su exigencia es entender esa presencia en cuanto tal, como negatividad efectiva, efectividad que brota como negatividad.

- Sólo al hacerlo, se niega al sujeto como figura única y sustancial. Esto es el trabajo negativo, el punto de rotación del movimiento del concepto, un flujo que no deja nada en reposo.

En este sentido, la penetración es un movimiento al interior de una noción de totalidad que entiende que el todo sólo se realiza a través de la distanciación, de la diferencia, donde los momentos parciales emergen y remiten fuera de sí mismos, disociándose, pero sin haber ninguna esencia por fuera de ellos. De este modo, se renuncia a todo afán de armonización y de continuidad, ya que en el conjunto complejo no hay continuidad sino un vuelco: "el proceso no transcurre por aproximación de diversos momentos, sino mediante un salto" (Hegel 1981: 82).

De este modo, la negación del sujeto proviene de un espíritu que sostiene la no detención, el no descanso en ninguna referencia sustantiva como fuente y origen de un sistema explicativo. Con esto hay una negación del sujeto, en cuanto: "al captar nosotros conceptualmente el recinto y los límites fijados a la subjetividad, al contemplar ésta como mera subjetividad, hemos traspuesto ya sus límites" (Adorno 1981: 20). Hay, entonces, una crítica frontal a esa descomposición lógica del conocimiento en sujeto y objeto, en cambio se los concibe como resultado de una mediación recíproca. Por su parte, la crítica se vuelve negativa en tanto moviliza y atraviesa esa concepción de polos en conceptos más bien rígidos y separados.

El sistema en Hegel refiere, no a un principio generador sino a una totalidad dinámica en donde las proposiciones se engendran unas a otras en virtud de su contradicción (Adorno 1981: 27). Esa fuerza de lo total es lo que lleva al trabajo negativo, a volver fluidos conceptos aislados, a especular con una cierta, al decir de Walter Benjamin (2009: 88) "dialéctica en suspenso".

Ahora bien, no se trata de encontrar desde Hegel una secuencia lineal de pensamiento. Tanto Adorno cuanto Luhmann al ganar el premio Hegel, lo que realizan son apreciaciones críticas, cuyas exigencias, bien descritas por Adorno consisten en entender que:

Lo que únicamente honrará a Hegel es, sin dejarse intimidar por el pavor ante la enredosidad poco menos que mitológica de un proceder crítico que aquella 
totalidad parece volver falso en todos los casos, en vez de otorgarle o denegarle méritos, persiga el todo tras el cual él mismo iba. (Adorno 1981: 16)

Ese todo, está compuesto sin duda por especulaciones lógicas negativas en torno a cómo es posible concebir la sociedad. Como Hegel mismo describe en su Ciencia de la Lógica:

La sociedad, en su complexión funcional, es un constitutum, un trozo de eso existente que se despliega a partir de lo absoluto, pero ni es independiente de él ni queda confundida con lo que designa, no hay que refundirla en una identidad, ya que la sociedad como todo no encuentra lo absoluto en ningún otro lugar que en la totalidad de la discordia, en la unidad con lo otro de uno. (Hegel 1981: 126)

La sociedad, por tanto, no contiene un mero existir, no tiene exterioridad ni es un mero hecho. Éste se constituye en la relación entre el espíritu y la sociedad, donde esta última es un concepto enfático que genera su propia movilidad. Así, ese flujo de pensamiento societal comprende un esfuerzo violento que caracteriza los conceptos hegelianos: las fatigas y los esfuerzos del concepto en su contradictorio recorrido. De allí que la unidad del sistema provenga de un poderío no reconciliado que se abre tensamente a la diferencia.

Por su parte, en el pensamiento de Adorno, la yuxtaposición coloca la dialéctica negativa como forma de mostrar el desgarro de sujeto y objeto como clave persistente de la no reconciliación.

- Tal vez es en su escrito contra el personalismo, donde es posible encontrar, entre otros, la emergencia de ese pulso disonante. Por eso permite desenmascarar tanto al sujeto, tanto al objeto como portadores de una ambición totalizante $\mathrm{y}$, a la vez, concebir la contradicción justamente como respuesta frente a la falsedad de la identificación total. Esto es clave para hacer emerger de otro modo, la relación entre individuo y sociedad (Adorno 1984: 274).

- Ahora bien, esta disonancia involucra la despedida a esa unidad en la pluralidad de las voces, mostrando en los trabajos del Beethoven tardío, esa renuncia proposital a la armonía que levanta un punto de fuga (Adorno 1964: 49).

- De allí que para poder yuxtaponer hay que diferenciar: "La diferenciación como procedimiento composicional significa articular los matices que señalan las diferencias cualitativas concretas entre fe- 
nómenos aparentemente similares" (Buck-Morss 1981: 208). Ahora bien, esa distinción se lleva a cabo por semejanza, cruzando su relación para observar la configuración, la constelación de la cual emergen. Como sostendrá Adorno (1964: 69): “Nunca las esencias estaban más marcadamente separadas que cuando más se acercaban unas a otras". En este sentido es un procedimiento composicional que significa articular los matices que señalan las diferencias. Los extremos se yuxtaponen para mostrar el carácter irreconciliablemente contradictorio de lo real, adentrándose de esta manera en el territorio vertiginoso de las negatividades efectivas.

De este modo, la yuxtaposición es una inversión:

Un primer eje a considerar es lo inverso al principio de diferenciación: yuxtaponer lo aparentemente no relacionado, los elementos no idénticos, revelando la configuración en la cual cuajaban o convergían. (Buck-Morss 1981: 209).

Por tanto, si se cruza la lógica de relación entre individuo y sociedad, asoma en Adorno un carácter claramente antihumanista:

El sujeto es la mentira, porque niega sus propias determinaciones objetivas con tal de poder mantener el absolutismo de la propia dominación. Sólo después de haberse desprendido de esa mentira y haber depuesto esa cáscara por sí mismo, comenzaría otra existencia. La monstruosidad ideológica de la persona es criticable inmanentemente, ya que lo sustancial que según esa ideología otorga a la persona su dignidad, no existe. (Adorno 1984: 275).

Sin embargo, esto no significa que se abrace a la sociedad y su función como una posibilidad de salida:

La dialéctica negativa no se detiene ni ante la coherencia de la existencia, la firme y humanista ipseidad del yo, ni ante su antítesis, igualmente endurecida, la función. Esta sirve a la determinación de la sociedad de modo análogo a la existencia de la ipseidad. (Adorno 1984: 278).

Lo que la monstruosidad pertinaz y represiva de la sociedad reproduce en la conciencia es lo contrario a la libertad y puede ser conjurado mostrando su determinismo. Colectivismo y humanismo se complementan mutuamente en la mentira. Sería hora de mandar a paseo la imagen del mundo absurdo después que de cifra de la nostalgia ha degenerado en lema de los fanáticos del orden. Pese a los cientistas de la sociedad actual, ésta no es abierta. (Adorno 1984: 282) 
Es decir, ya sea que se instale el individuo ante la sociedad como un fundamento o ésta como su objeto inconmensurable "un destino ciego se apodera de la comunicación entre ambos" (Adorno 1973: 161). La comunicación requiere, por tanto, un proceso de reconocimiento donde lo diferente sea compartido (Adorno 1973: 145.)

Como se puede observar, la interpenetración genera distinciones no sólo en relación a una teoría tradicional sino que resulta clave entender sus aprecios y divergencias con esa raíz hegeliana de pensamiento negativo que tanta diversidad logra impulsar en la contemporaneidad, pero que no se reconoce en algunos ámbitos como las políticas públicas, tan marcados hasta ahora por un pensamiento que no sólo proviene de una suerte de teodicea del mercado (recordando a Weber) sino de una lógica formal que no sabe trabajar con la contradicción sino con la supremacía de una episteme pre hegeliana.

\section{EL GIRO EN LA NOCIÓN DE INTERPENETRACIÓN EN LUHMANN}

En Luhmann la noción de interpenetración trata de una relación entre sistemas que pertenecen recíprocamente uno al entorno del otro. Por tanto, no es algo que ponga foco en el sistema pero tampoco en lo que existe entre los sistemas. No hay una zona fronteriza, un espacio de interacción, un tercero, una brecha o un conjunto de superposiciones (Luhmann 1998: 200). Lo anterior da lugar a una serie de condiciones de posibilidad para asumir este concepto:

- Se requiere asumir de parte a parte la superación del sujeto como principio explicativo. Pero sujeto no sólo en cuanto sistema psíquico, sino como ese espacio declarado desde una positividad. Sólo así se hace emerger un giro. "El centro de la diferencia consiste en que para la tradición humanista, el ser humano se encuentra en el centro y no fuera del orden social, como elemento de la sociedad misma. Cuando al ser humano se le consideraba como último elemento de la sociedad que no podía ser descompuesto ulteriormente" (Luhmann 1998: 199). Ahora bien, "si se considera que el ser humano es parte del entorno de la sociedad, cambian las premisas sobre las cuales se cuestiona la tradición, es decir, las premisas del humanismo clásico" (Luhmann 1998: 201). 
- En la interpenetración el sistema receptor ejerce también una influencia retroactiva sobre la formación de estructuras del sistema penetrador, interviniendo, por tanto, de dos maneras, desde el interior y desde el exterior. Debido a ese reforzamiento de las dependencias, es posible obtener un mayor grado de libertad, por tanto, la interpenetración individualiza más que la penetración. "Precisamente en este sentido, hay interpenetración cuando ambos sistemas se posibilitan aportar al otro su propia complejidad preconstruida" (Luhmann 1998: 202).

- Al mismo tiempo, al entender la complejidad como coacción de selección, es posible poner a disposición del otro esa complejidad inaprensible, aportar ese desorden. De esa forma, se impulsa mayor posibilidad de selectividad interna y se produce mayor autonomía. Para entender lo anterior hay que remarcar la importancia que el aspecto central de esta concepción es que "los sistemas que se interpenetran permanecen como entorno uno para el otro. Por eso es válido formular también que los sistemas psíquicos proveen a los sistemas sociales de suficiente desorden y viceversa" (Luhmann 1998: 202). Entonces, la interpenetración supone una combinación de orden y desorden, eso que ya Heinz Von Foerster (1991) denominaba el "principio de ruido". De allí que las políticas públicas en tanto parte de sistemas sociales surgen de los intentos de comunicación, de los ruidos producidos por los sistemas psíquicos (Luhmann 1998: 203).

- Sin embargo, las acciones humanas no pueden concebirse como un elemento del sistema, las acciones no se dan sin sustrato; lo que significa que ellas ya están permeadas por el propio sistema que las engendra mediante una "capacidad de enlace" (Luhmann 1998: 203). Profundizar en la forma en que acciona esta capacidad supone ahondar los mecanismos por los cuales se producen las selecciones. En este sentido "las acciones abastecen las condiciones de la combinación social" (Luhmann 1998: 203). Por tanto, los sistemas que se interpenetran aportan sus elementos pero hay que entender sobre todo que les confieren cada vez una "selectividad y una capacidad de conexión distintas, un pasado y un futuro distintos" (Luhmann 1998: 203). De allí que los que participan seleccionan posibilidades y producen consecuencias que son distintas. 
- De esta forma, la interpenetración aclara y potencia los mecanismos de doble contingencia, ya que viabilizan sus condiciones de posibilidad. Se trata de mutua factibilización, de posibilitar un proceso de comunicación. Ahora bien, los sistemas que recurren a estas posibilidades son aquellos que son capaces de "resistir las inestabilidades más altas y los cambios estructurales más rápidos, exponerse más fácilmente a las causalidades y aligerar con ello, sus sistemas de regulación. Esto es posible cuando se entiende la complejidad y la interpenetración correctamente, es decir, cuando se las entiende como coacción de selección que se incrementa y como condicionalidad abierta precisamente de esta coacción" (Luhmann 1998: 205).

- Lo interesante es que esta concepción permite entender que el aporte de un sistema es un ramillete de recursos, informaciones, formas de selección. Pero esto no se puede observar como una posibilidad de rendimientos crecientes sino como "un plexo de constitución. Cada sistema estabiliza su propia complejidad" (Luhmann 1998: 205). Así, la estabilidad surge de un sistema de ajustes continuos, de la riqueza de posibilidades que involucra la modificación de las condiciones, promoviendo, a la vez, cambio y continuidad. De esta forma "cada sistema estabiliza sus propias inestabilidades" (Luhmann 1998: 205).

- Ello requiere de un sistema de enlace: "el enlace es la fijación del sentido de las posibilidades abiertas mediante la estructura de un sistema emergente" (Luhmann 1998: 208). En esto hay una dimensión temporal que considerar. Cada acontecimiento, cada selección, provoca un efecto que excluye posibilidades y abre otras. Existe, de esta forma, una acción negativa a considerar, la cantidad de exclusiones que configuran el sustrato desde donde se selecciona. Por esto, la fórmula para entender la interpenetración "es la diferencia y no la unidad; y no se refiere al ser de los sistemas sino a su operación reproductiva" (Luhmann 1998: 217).

- Por último, las relaciones de interpenetración son relaciones sistema/entorno, es decir, relaciones de un sistema con un entorno específico de sistemas interpenetrantes. La tesis establece que los aumentos graduales de complejidad de los sistemas sociales, transforman las relaciones de interpenetración, las diversifican y las relacionan menos directamente con su transcurso natural (Luhmann 1998: 235). Por tanto, existe en esta configuración conceptual enormes potencialidades para hacer aparecer cuestiones antes improba- 
bles, "creando formas y deslindamientos que, por su parte, produzcan nuevas consecuencias" (Luhmann 1998: 235).

\section{APORTES DEL CONCEPTO DE INTERPENETRACIÓN A ALGUNAS LÓGICAS DE DEBATE EN LAS POLÍTICAS PÚBLICAS.}

La derrota de la metafísica, de un pensamiento estructural, convencional, es requerido imperiosamente a todos quienes busquen acceder a estos conceptos: penetración, yuxtaposición, interpenetración.

Hay que matar al sujeto: es Hegel quien está convencido que la época de la Ilustración que culmina con Kant y Fichte no ha erigido en la razón sino un ídolo. Al sustituir equivocadamente la razón por el entendimiento y desplazando la razonabilidad a una razón instrumental se ha elevado a lo absoluto algo finito: "El entendimiento, al fijar lo infinito, lo opone absolutamente a lo finito y la reflexión que se había elevado a la razón al superar a lo finito, se rebaja otra vez al entendimiento al fijar la obra de la razón en una oposición" (Hegel 1969: 47).

Lo anterior supone, una tarea de enorme envergadura: el desvanecimiento de los referentes metafísicos para observar el orden/desorden de las relaciones mundanas. Evitar que "la metafísica se nos cuele por la ranura" (Adorno 1984: 98), significa abrirse a la interpenetración, respetando con gentileza sus exigencias lógicas, despliega un plexo de sentido y nuevas posibilidades políticas. No tanto por saber de antemano adonde nos lleva, sino permitiendo observar con toda claridad de donde nos saca.

En el nivel de la teoría general de sistemas se constatan tres variables: "el relajamiento de los lazos internos y los enlaces de los sistemas que se interpenetran, la aportación específica de las interpenetraciones y la producción de efectos por acumulación" (Luhmann 1998: 358). En términos del relajamiento de los lazos internos en los sistemas sociales, es posible apreciar que las formas de vivir están menos tipificadas por tradiciones sociales que obligan interiormente. A las referencias de cuna y clase, se le adicionan no sólo el estatus adscrito sino el adquirido. De allí que crezca la participación en ajustes sociales y, a su vez, la desconfianza y el aislamiento de las formas tradicionales de la adscripción. Esto contribuye a un panorama de mayor complejidad. Por su parte, la acumulación de efectos se presenta como agregaciones inesperadas cuyos efectos rebasan los umbrales determinados. La falta de apoyo en estructuras permanentes del sistema social, entre otras sus políticas públicas, agudizan el problema y reducen las posibilidades de 
encontrar soluciones para nadar siguiendo las corrientes temporales y para cambiar de dirección. Entonces, una forma de autobservación funcional puede aportar a entender el movimiento como categoría de inestabilidad/estabilidad, donde la fijación de metas es siempre una coartada para no ver la razón para el movimiento perpetuo. En este sentido, los diversos movimientos no son necesariamente acontecimientos comunicacionales. Reaccionan ante las perturbaciones, no en relación con el entorno, sino con el círculo de comunicación mismo (Luhmann 1998: 362). De allí que resulte válido entender la crítica como una continuidad de la comunicación y no como una conciencia crítica de lo existente.

La continuación comunicativa, abre la posibilidad de interpenetraciones y muestra que la sociedad actual comparada con los procesos históricos, desestabiliza las estructuras y aumenta considerablemente el potencial de la negación. Será entonces menos importante saber si se articula más desde posiciones de poder, del derecho o desde el contexto de los movimientos sociales. (Luhmann 1998: 362)

Lo interesante es observar los mecanismos por los que se introduce improbabilidad y se sostiene la comunicación de lo diferente.

Lo anterior puede ser llevado a diversos ámbitos de enfrentamiento de controversias en políticas públicas. Acá se ofrecen dos ejemplos de aportes específicos:

- La observación de las lógicas de conservación y cambio en la mantención de ciertas dicotomías naturalizadas: la oposición entre pobreza y desigualdad. Desde la oposición de crecimiento o redistribución no es posible observar posibilidades de intercambio, aunque existen numerosas evidencias en estudios e intervenciones sociales que la separación radical no conlleva a buenos resultados, porque se da lugar a efectos pendulares que siempre van a frenar las oportunidades de interpenetración, al evitar la apertura comprensiva de la complejidad sistémica de esos procesos. Es más, al leer esto como crisis radical y anuncios de caos: "se perderá todo lo alcanzado, se calentará la economía con gravísimas consecuencias, crecerá el desempleo, con ello la delincuencia y el descontento, se retirarán las inversiones". O por otra parte, en la desconfianza radical: "ya ven la izquierda también roba, la corrupción es transversal, la política es inservible, los mecanismos de diálogo están todos cooptados, hay que anular al capital en todas sus formas, las instituciones están en el suelo, nos van a engañar siempre con promesas de cambios y ellos no serán nunca suficientes, son 
oportunistas y no sustantivos". De esta forma la lógica de lo aparentemente opuesto, opera como reforzamiento y reduce las probabilidades de superación y desplazamiento de sistemas de observación. La ampliación del miedo, como ya sostenía Norbert Lechner (1988: 95 y ss.) en sus "Patios interiores de la democracia" actúa como un detonante enunciativo que provoca lo que predice. En esto la agenda y los padrones de énfasis comunicacional en los medios se torna relevante de examinar.

- Muchas veces en las formas narrativas, las lógicas que las posibilitan no se visivilizan. Una contribución relevante es entender las posiciones discursivas: frente al discurso de Bachelet que inaugura su segundo mandato "Chile tiene un solo un gran adversario y eso se llama desigualdad, y sólo juntos podremos enfrentarla". A él se le opone una forma de observación discursiva largamente reiterada: "Los pobres no pueden esperar, la desigualdad sí" (mann \& Hinzpeter 2000). De esta forma, se entiende que los chilenos demandan dos cosas: igualdad y crecimiento económico pero a la hora de elegir prefieren desarrollo económico. Su primera aspiración sería salir de la pobreza: "por eso Chile no puede darse el lujo de poner en riesgo la creación de empleo y el crecimiento" (Lehmann \& Hinzpeter 2000). Los pobres no pueden esperar, la desigualdad sí supone que es el crecimiento el que llevará a la mayor igualdad a mediano plazo. Para esta lógi$\mathrm{ca}$, incluso momentos de igualdad y crecimiento se transforman en un trade off: "Poner en riesgo el crecimiento por una mayor igualdad en un populismo imprudente. La gente quiere ir más rápido, pero la prudencia es ir según el crecimiento" (Lehmann \& Hinzpeter 2000). El que las tres mandatarias no sepan leer esto, provocaría aún mayor descontento.

- Sin embargo, hay una forma diversa de entender este malestar por asincronías entre subjetividad y modernización. Existe, por un lado, el ritmo acelerado de las transformaciones económicas. En términos estructurales, el rasgo sobresaliente de la época es la mayor diferenciación de "sistemas funcionales" con "reglas del juego" específicas (PNUD 1998). 
FIGURA 2. COORDENADAS DE LA MODERNIDAD SEGÚN EL PNUD

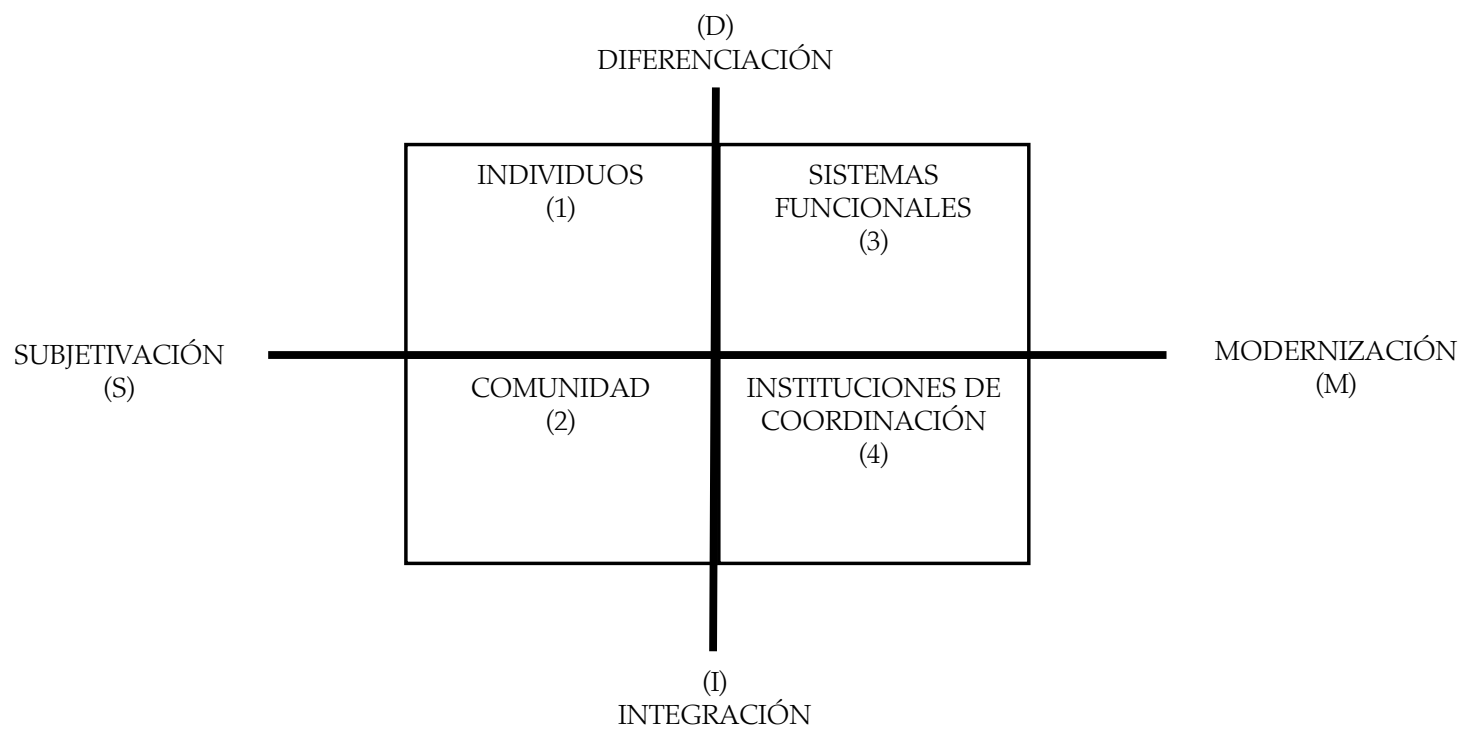

FUENTE: PNUD (1998)

- Es decir, la evolución no ha seguido el ritmo de "la gente" sino que la aceleración de los sistemas de integración como son el Estado y el mercado, en sus instituciones de coordinación, han impactado fuertemente a los procesos de producción de subjetivación. La observación de las secuencias temporales: Del ritmo rápido de los monopolios. De los desplazamientos del principio de solidaridad al de responsabilidad individual. De la desprotección concebida con las privatizaciones de sistemas sociales de salud, de previsión social, de educación. Del ritmo lento de las aspiraciones civiles en Chile: 104 años el divorcio, 38 años la ley de inmigración, 65 años el voto femenino. De la metáfora institucional de país: "Chile no está preparado para..." De esas lógicas que ya Albert O. Hirschman (1991) denominaba las "retóricas de la intransigencia". Por tanto, es posible concebir que la paradoja proviene por saturación de un proceso neoliberal que se niega a retroceder y de un avance en la visibilización, al volver a poner sobre la mesa sus naturalizaciones violentas. Abrirse a concebir los ruidos y hacer el desencanto fructificar contiene potencialidades de inestabilidad/estabilidad.

- De las formas de observación de lógicas de la repetición en la medición de los éxitos/fracasos en las políticas públicas. Es interesante como esto condiciona las posibilidades de coordinación funcional, porque las restringe en sus formas de mirar y, por tanto, reduce posibili- 
dades de interpenetración. En las actuales formas de medición que se condicen con los programas de transferencia de renta ha primado una observación de la demanda. De allí que las evaluaciones de impacto tienen tres condiciones: se hacen después, la hacen expertos externos, contienen una gran cantidad de indicadores. Asimismo, el foco a evaluar lo constituyen esa cantidad de participantes que son enunciados como "poblaciones en riesgo, sujetos vulnerables, familias disfuncionales, barrios peligrosos, jóvenes infractores de ley".

Para girar lo anterior y abrirse a otras posibilidades actualmente se desarrolla un proyecto Fondef-Conicyt Chile (Matus 2015) que busca efectuar una evaluación sobre la oferta para anticipar el nivel de calidad del programa, seleccionando cuatro variables mínimas, identificar la complejidad del contexto en las condiciones de calidad en que se lleva a cabo la intervención, considerar la heterogeneidad de los fenómenos sociales, e impulsar una toma de decisiones oportuna. La apuesta es que la articulación de esas dimensiones, en un sistema tecnológico integrado y de fácil manejo para los propios equipos profesionales, dará como resultado una innovación que incremente la efectividad de la evaluación de los programas sociales.

Es decir, se busca mostrar que la precariedad se instala en las condiciones de calidad de la oferta de los servicios, en sus brechas para leer la complejidad heterogénea de sus contextos; y que la vulnerabilidad sistémica pone en peligro a las personas y comunidades que participan en esos programas, ya que un hallazgo reiterado es observar que a medida que la complejidad aumenta, las condiciones de calidad de la oferta disminuyen. Esto posibilita poner en marcha un proceso de autobservación de los propios equipos de trabajo y sus organizaciones que, potenciado por la posibilidad de la medición rápida y sistemática, contenga la construcción de planes de mejora donde exista una real apropiación de los ajustes y de las formas de relación con los participantes de ese programa. De esta forma, es posible mensurar a priori los flujos de riesgo de la inversión, pensar mejor los mecanismos de oferta, potenciar equipos de trabajo y apoyar un manejo más ajustado y oportuno de toma de decisiones que mejoren gradualmente la calidad y los estándares en los que se miden y observan resultados.

Así, la interpenetración se observa como un portal para pensar no sólo en lo peor o lo mejor sino en abrir improbabilidades y poder postular 
combinaciones donde la enunciación de la crisis puede contener retóricas no dicotómicas, la evaluación puede ser antes, postular que lo uno puede ver lo otro, que menos puede ser más. Observar que la contingencia funciona como unidad de la diferencia moderna (Mascareño 2012: 57). Entender que las políticas públicas presentan una tarea pendiente que puede ser postulada como un déficit de observación y que sus análisis han tendido a prescindir de las teorías generales de la sociedad y que por tanto se encuentran en la paradoja de querer aportar a la agenda social sin sistemas lógicos adecuados para pensar la sociedad en que vivimos (Matus 2012: 205). Negar para salir, destruir para construir, hacer un elogio de la sombra, en su luminosidad. Ya que en la interpenetración vive la interrogante sobre el modo de nombrar y de mirar en una sociedad funcionalmente diferenciada. $\mathbb{R M}$

\section{REFERENCIAS}

Adorno, T. (1964). "Maurice Ravel." En Moments Musicaux: Neu gedruckte Aufsätze 1928-1962. Frankfurt aM: Suhrkamp.

Adorno, T. (1973). Epilegómenos Dialécticos. En Consignas. Buenos Aires: Amorrortu.

Adorno, T. (1981). Tres estudios sobre Hegel. Madrid: Taurus.

Adorno, T. (1984). Dialéctica Negativa. Madrid: Taurus.

Arendt, H. (1999). Eichmann en Jerusalén: Un estudio sobre la banalidad del mal. Barcelona: Lumen.

Baudrillard, J. (1995). La transparencia del mal: Ensayo sobre los fenómenos extremos. Barcelona: Anagrama.

Buck-Morrs, S. (1981). Origen de la dialéctica negativa. México DF: Siglo XXI.

CEPAL (2006). La protección social de cara al futuro. Consultado el 25 de mayo, 2014, desde http://www.cepal.org

Coady, D., Grosh, M., \& Hoddinott, J. (2004). Targeting of Transfers in Developing Countires. Consultado el 25 de mayo, 2014, desde http://www.worldbank.org

Cohen, E. \& Franco, R. (2006). Transferencias con corresponsabilidad. México DF: FLACSO.

Hegel, G.W.F. (1969). La ciencia de la lógica. México DF: FCE.

Hirschman, A. (1991). Retóricas de la intransigencia. México DF: FCE.

PNUD (1998). Informe de Desarrollo Humano en Chile: Las paradojas de la modernización. (Informe dirigido por Norbert Lechner). Santiago de Chile: PNUD.

Irarrazaval, I. (2004). Sistemas únicos de información sobre beneficiarios en América Latina. Consultado el 25 de mayo, 2014, desde http://www.iadb.org/publications

Kliksberg, B. (2011). Escándalos éticos. Buenos Aires: Temas.

Lechner, N. (1988). Los patios interiores de la democracia. Santiago de Chile: FLACSO.

Lehmann, C. \& Hinzpeter, X. (2000). Punto de referencia $n^{\circ}$ 53: Los pobres no pueden esperar la desigualdad sí. Santiago de Chile: CEP. 
Luhmann, N. (1998). Sistemas sociales: Lineamientos para una teoría general. Bogotá: Anthropos, CEJA.

Mascareño, A. (2012). Contingencia como unidad de la diferencia moderna. En: H. Cadenas, A. Mascareño, y A. Urquiza (Eds), Niklas Luhmann y el legado universalista de su teoría. Aportes para el análisis de la complejidad social contemporánea (pp. 57-80). Santiago de Chile: RIL.

Matus, T. (2012). Observar la complejidad: Un desafío a las políticas públicas. En: H. Cadenas, A. Mascareño, y A. Urquiza (Eds), Niklas Luhmann y el legado universalista de su teoría. Aportes para el análisis de la complejidad social contemporánea (pp. 215-219). Santiago de Chile: RIL.

Matus, T. (2015)- Proyecto Fondef: Innovación Social Efectiva. Construcción de una batería de instrumentos cuantitatioos para mensurar la calidad de los programas sociales. IDeA IT13I10020.

Serrano, C. (2005). La política social en la globalización. Programas de protección social en América Latina. Serie Mujer y desarrollo 70. CEPAL. Consultado el 25 de mayo, 2014, desde http://www.cepal.org

Weber, M. (2002). Economía y sociedad. México DF: FCE.

Durkheim, E. (2007). La división del trabajo social. México DF: Colofón.

Von Foerster, H. (1991). Las semillas de la cibernética: Obras escogidas (M. Pakman, Ed.). Barcelona: Gedisa.

\section{SOBRE LA AUTORA}

Teresa Matus es Trabajadora social por la Universidad de Concepción. Doctora en Sociologia IUPERJ Rio de Janeiro y Doctora en Trabajo Social UFRJ. Actualmente es la Directora de la Carrera de Trabajo Social de la Universidad de Chile, Directora del Núcleo de Investigación en Sistemas Sociales y Complejidad Sociocultural (NISS) de la Facultad de Ciencias Sociales, Universidad de Chile y Socia fundadora de la Sociedad Chilena de Políticas Públicas. Entre sus últimas publicaciones se destacan: Observar la complejidad: Un desafío a las políticas públicas (En: Niklas Luhmann y el legado universalista de su teoría. Santiago de Chile, 2012); Investigando en Red, Estudios Internacionales en Trabajo Social (junto a Juana Arias Rojas, Carolina Muñoz Guzmán y Malvina Ponce de León. Santiago de Chile, 20099.

\section{CONTACTO}

Departamento de Trabajo Social

Facultad de Ciencias Sociales, Universidad de Chile

Avenida Ignacio Carrera Pinto 1045, Ñuñoa

Santiago, Chile

CP: 6850331

teresamatus@u.uchile.cl

Recibido: Junio 2015

Aceptado: Agosto 2015 